\title{
Isothermal Modeling of Perforated Duct Diffuser (PDD)
}

\author{
Peyman Raphe ${ }^{1, *}$, Hachimi Fellouah ${ }^{1}$, Mohamed Ameur ${ }^{2}$ \\ ${ }^{1}$ Department of Mechanical Engineering, Université de Sherbrooke, Sherbrooke, QC, Canada \\ ${ }^{2}$ NAD Klima, Sherbrooke, QC, Canada \\ *peyman.raphe@usherbrooke.ca
}

\begin{abstract}
In this study, a numerical model has been developed to characterize a room air distribution using the perforated duct diffusers (PDDs). The study is limited to the isothermal mode with no consideration of buoyancy forces, wall radiations, convection flows, etc. The numerical simulations have been compared against experimental measurements (not shown here), and the most proper index was used to indicate ventilation performance. The CFD simulations were performed by using the commercial ANSYS Fluent software. The results indicate an improvement in the air distribution inside the room using PDD. Furthermore, by analyzing different comfort indices such as terminal velocities, the discomfort zones have been determined in the occupant zone.
\end{abstract}

Keywords-isothermal; numerical modeling; perforated duct diffuser; air ventilation; air distribution pattern.

\section{INTRODUCTION}

The unfavorable atmospheric conditions would be destructive and unhealthy for the people in the long term in the highly crowded zones. As a result, HVAC (Heating, Ventilation, and Air-Conditioning) systems are gained a higher weight on predesign considerations. Ventilation systems are categorized as air or heat ventilation [1]. The principal purpose of ventilation, which could be in the shape of mechanical or natural ventilation, is to dilute and displace the air in a specific area [2-3]. HVAC designers mainly deal with a wide variety of local and general requirements. However, low energy consumption and ventilation effectiveness should be noticed for designing ventilation systems.

By the end of the 70s, the principle of sick building vs. healthy buildings has prevailed as a result of the predicament of energy in tighter buildings. The lack of sufficient outdoor air ventilation causes the sick building syndrome (SBS) and lower performance of occupants. After the 90s, the improvement of indoor air quality (IAQ) was widely highlighted, due to the close relationship of occupants' health and thermal comfort [3]. ASHRAE Standard 62.1 suggested two procedures for IAQ improvement by ventilation: a) Ventilation air procedure, and b) Indoor air quality procedure [2].
Ventilation devices exist in different shapes that each has its advantages and disadvantages in comparison. Most of these systems ventilate the fluid into the zones via so-called diffusers. Many authors examined different geometries in their works. Liu et al. [4] used the rectangular ceiling diffuser and spherical nozzles to compare mixing, stratified air, and air curtain ventilation. Sajadi et al. [5] investigate the effects of swirling diffusers at different angles and slot geometries.

Perforated duct diffusers as a practical type of these devices follow the concept of semi-continuous ventilation. In this context, the fluid distributes in the domain from lots of holes. The uniform air distribution with a low draft is the main advantage of this type of diffusers [6-7]. Anyhow, these diffusers should be characterized before apply. The majority of the previous studies are allocated to the simulations of the conventional diffusers, and yet there is a not considering area for the PDD. Moreover, the diffusers are compared from a different point of view using indices of performance, comfort, contaminant removal, and energy consumption.

\section{COMFORT INDICES}

The final goal of the diffuser design is to increase air quality through proper air distribution. In this context, numerous indices have been defined by the Standards (ASHRAE, ISO, EN, etc.) to evaluate the performance of the HVAC systems, especially ceiling air diffusers' thermal comfort, energy consumption, noise level, ventilation effectiveness. However, there are no explicit standards and guidelines allowing the evaluation of the performance of the air distribution using PDDs.

In this study, the results of the simulations depicted according to the requirement of the ASHRAE Standard. Three parameters, namely Throw, Drop, and Spread (Fig. 1), have been used to evaluate the air distribution in the room. Technically, the throw is the distance from the center of the face of the diffuser's outlet to a point where the velocity of the air stream reduces to a particular amount, usually $150 \mathrm{fpm}$ $(0.75 \mathrm{~m} / \mathrm{s}), 100 \mathrm{fpm}(0.50 \mathrm{~m} / \mathrm{s})$, or $50 \mathrm{fpm}(0.25 \mathrm{~m} / \mathrm{s})$. These velocities refer to Terminal Velocity and known as T150 (T0.75), T100 (T0.50), or T50 (T0.25), respectively. Drop is the vertical spread of the airstream due to entrainment of room 
air, and the buoyancy effect due to the density discrepancy between the total air package and the surrounding primaryroom air lead to the drop of cool total air [8]. Spread defined as the divergence of the air stream in a horizontal or vertical plane, which is a function of the diffuser's outlet geometry. However, these parameters are valid in isothermal mode only. In the case of heating or cooling mode, the requirements of the air distribution are referenced in the ASHRAE 55 and ASHRAE 62.1 standards.

\section{NUMERICAL SIMULATION}

\section{A. Geometry}

A duct diffuser has been modeled at a room with a dimension of $3964 \times 3050 \times 2430 \mathrm{~mm}^{3}$, Fig 2, using ANSYSFluent. There is a $600 \times 150 \mathrm{~mm}^{2}$ outlet at the ceiling corner. The distance from the centerline of the diffusers to the ceiling and side walls equal to $406 \mathrm{~mm}$ and $1525 \mathrm{~mm}$, respectively. The side window and walls are isolated to meet the isothermal conditions. The materials which are used for diffusers and walls have a very low friction coefficient. Furthermore, it's assumed that the diffusers are fixed without vibration and movements.

\section{B. Initial and Boundary Conditions}

The single-phase fluid (air with constant density) distributes inside the domain with $2.4 \mathrm{~m} / \mathrm{s}$ initial velocity and $101325 \mathrm{~Pa}$ ambient pressure, Fig. 2. The entire surfaces are isolated to maintain the isothermal condition $(\mathrm{T}=295 \mathrm{~K})$. The stationary walls have a no-slip boundary condition with zero heat flux and follow standard roughness models.

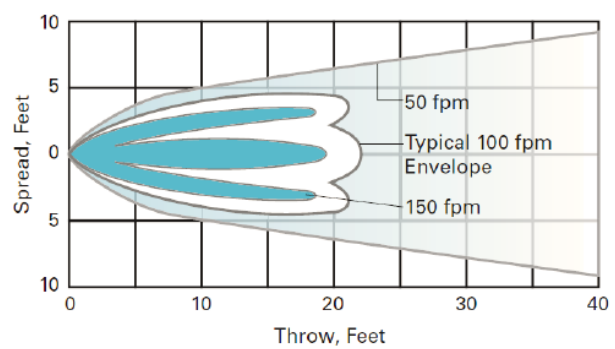

Figure 1. Ventilation parameters [8].

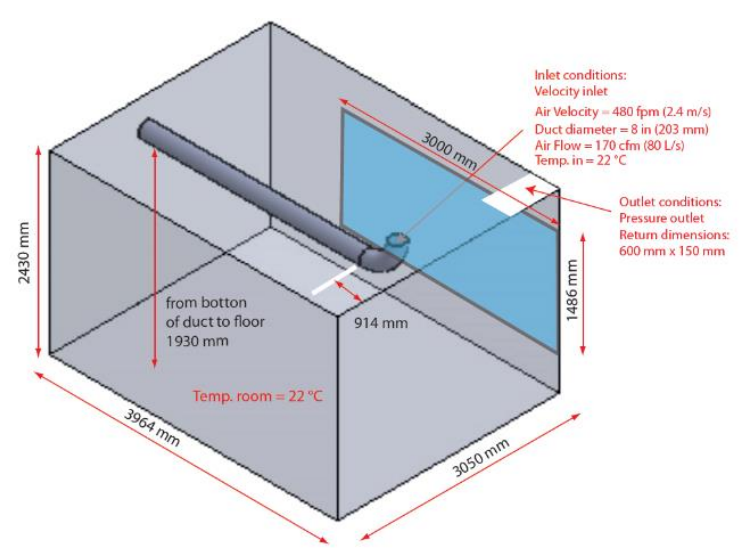

Figure 2. Test room layout.

\section{Mesh Generation}

According to the geometry of the problem, the size of the largest (Fig. 3-a) and the smallest (Fig. 3-b) phenomenon has a large discrepancy, and this is the main challenge of duct diffuser simulation. Due to the fact, one can observe large and small velocity gradients at the same time in different places [9]. Though, the mixing pattern of the air inside the occupant zone is the principal phenomenon to study.

Mesh sensitivity analysis was performed for different mesh element sizes, and finally, 2394470 unstructured linear elements with 0.84 element quality has been applied for this simulation.

\section{Turbulent Model}

The flow regime is 3D, incompressible, steady, viscous, isothermal, and fully turbulent. With constant density and temperature hypothesis, Navier-stokes and conservations equations solved at the domain using ANSYS-Fluent 2019-R3. Air distribution patterns compared for eight different turbulence models include Spalart-Allmaras, k- $\varepsilon$ (Standard, RNG, and Realizable), k- $\omega$ (Standard, GEKO, and SST), and Transition SST $\omega$. The pressure-based solver was used for solving governing equations considering the effects of gravity acceleration. Wherever it was necessary, enhanced wall treatment has been applied.

\section{E. Validation}

The results have been validated against the experimental measurements (not shown here and research work will be published) performed at the NAD Klima company to examine the accuracy of the numerical simulations. The k- $\varepsilon$ Realizable turbulence model provides the highest adequate results. Moreover, the temperature measured continuously at different locations to assure the isothermal condition.

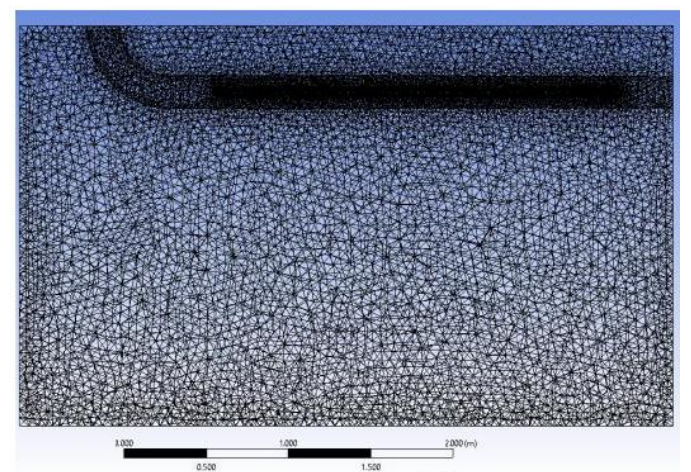

(a)

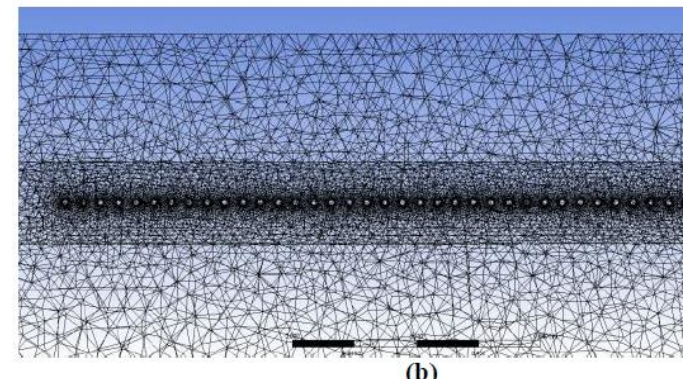

(b)

Figure 3. Mesh details. a) Domain; b) Holes. 


\section{F. Error and Uncertainties}

The simplification assumptions of numerical models lead to errors and uncertainties at final results. Meanwhile, it's possible to rectify these errors by applying sensitivity analysis methods, proper convergence criteria, solution methods, etc.

The mesh type and element numbers have high impact on the final errors. The $\mathrm{y}^{+}$amount has been calculated for different turbulence methods to define appropriate mesh element size and wall functions.

The turbulence methods are another sources of the errors. Generally speaking, the gradient of mean quantities in the turbulence flow is higher than laminar flow. The lower order of schemes, the higher numerical diffusion, and the lower result accuracy would be attained, especially for the convection terms. In the present study, the second-order schemes have been applied, and the 1e-04 convergence criteria set for the residuals [10].

\section{RESULTS}

As previously stated, the injected air from the perforation are subjected to the coincidences, and in consequence, parameters like Throw, Drop, and Spread (TDS), do not follow the conventional shape. Fig. 4 illustrates the terminal velocities at the so-called diffuser holes' plane. A portion of the fresh air, which passes alongside the duct, exits from the holes in

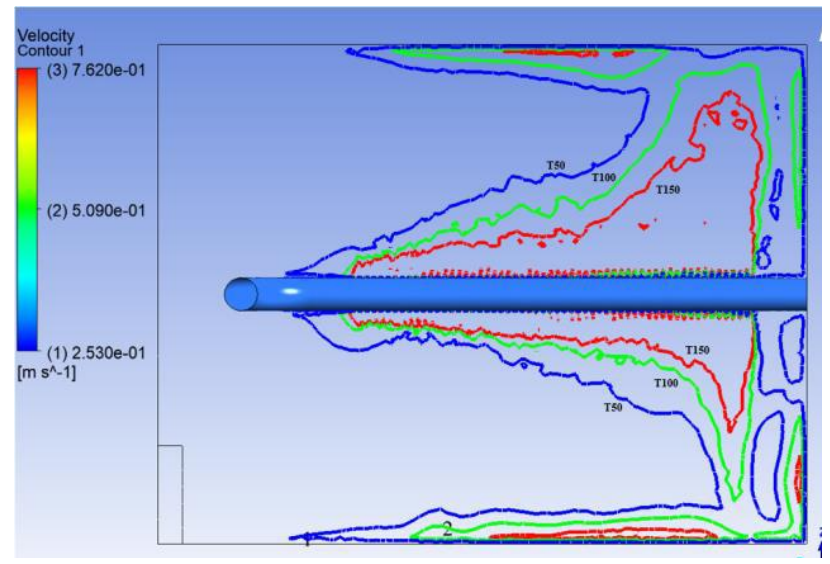

Figure 4. Terminal velocities at diffuser holes plane.

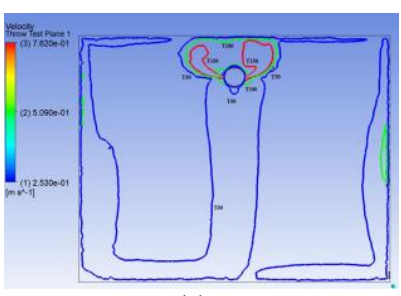

(a)

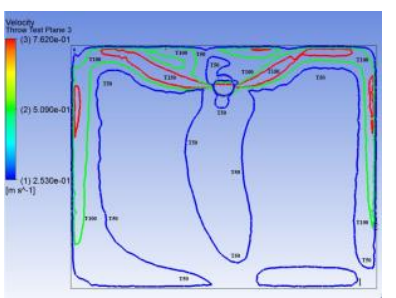

(c)

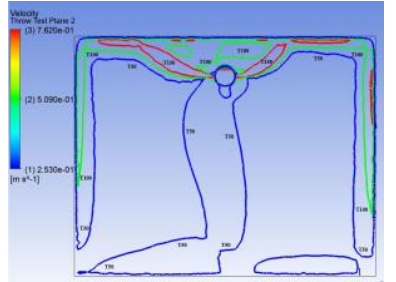

(b)

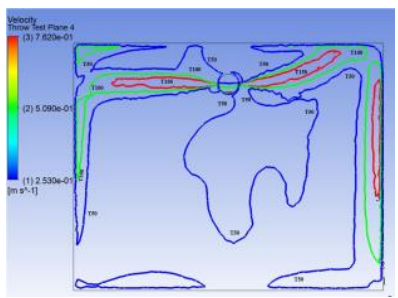

(d)
Figure 5. Terminal velocities $2 \mathrm{D}$ contours at test planes. a) $\mathrm{X}=-0.926$, b) $\mathrm{X}=-1.578, \mathrm{c}) \mathrm{X}=-2.174$, and d) $\mathrm{X}=-2.822$. different directions. The angle between the injected air and the flow direction increases alongside the duct. This fact leads to more air particle collisions at the early holes and inclination of the flow.

Fig. 5-a to 5-d show the variation of the terminal velocities, and TDS consequently, at four different planes that were used for experimental measurements, at the test room. These planes have been located at distance 926, 1578, 2174, and $2822 \mathrm{~mm}$ from the inlet center, respectively. The shapes of these 2D contours depend on the inlet air velocity, duct geometry, ventilation mode, and the number of the holes.

From literature, the occupant (breathing) zone is the region within an occupied space between planes 3 in and 72 in (75 $\mathrm{mm}$ and $1800 \mathrm{~mm}$ ) above the floor and more than $2 \mathrm{ft}$ $(600 \mathrm{~mm})$ from the walls or fixed air-conditioning equipment $1 \mathrm{ft}$ from each side [ $2 \& 8]$. The $3 \mathrm{D}$ contour of the points that have a velocity magnitude higher than $0.25 \mathrm{~m} / \mathrm{s}$ at the occupant zone distinguishes the discomfort zones, Fig. 6.

Another important fact that should be considered is related to the selection of the performance indices. For instance, indices like Effective Draft Temperature (EDT), underestimate or overestimate the discomfort zones. Even though these indices used by numerous authors [10-12], Fig. 7 shows the incapability of the EDT index to estimate the correct discomfort zones.

\section{CONCLUSION}

The main rule of HVAC systems is to provide favorable indoor air quality for the people. The total performance of these systems, which indicates their energy consumption level, links to the performance of the subsections like a diffuser. Perforated duct diffusers find their applications at new designs, specifically for the ventilation of the vast space.

To compare the performance of these diffusers, one has to characterize them either numerically or experimentally. Since, there is no previous work on PDDs, a uniform circular duct with vertical perforation on both sides has been modeled, in the

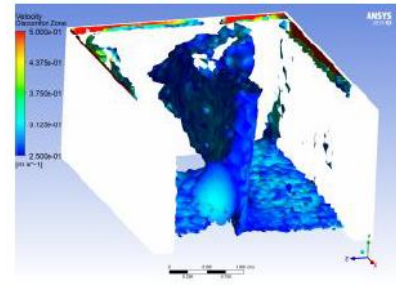

(a)

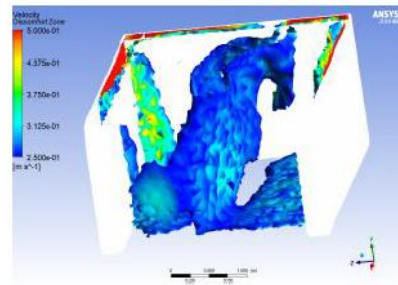

(b)
Figure 6. 3D contour of the terminal velocities (discomfort zone) at the occupant zone. a) Right side, and b) Left side.

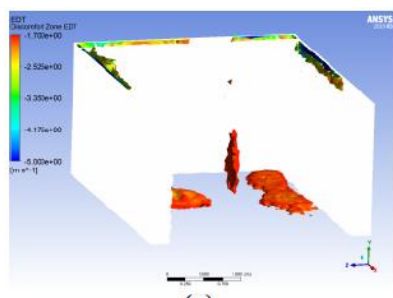

(a)

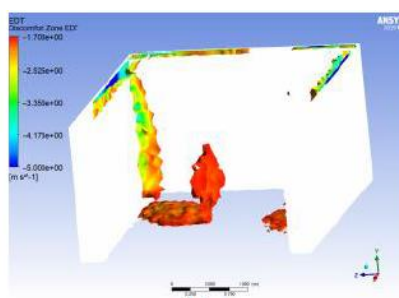

(b)
Figure 7. 3D contour of the EDT (discomfort zone) at the occupant zone. a) Right side, and b) Left side. 
isothermal ventilation mode. In this context, the diffuser has been nominated to circulate the air in the region with the actual size, i.e., full-scale simulation. A bunch of sensitivity analyses have been performed assuring the accuracy of the results. Finally, the number of 2394470 unstructured linear elements have been solved by the $\mathrm{k}-\varepsilon$ Realizable turbulent model to capture the air distribution pattern inside the room.

Terminal velocities distinguish the borders of the discomfort zones following the standards and local regulations. 2D contours are used to determine the TDS parameters, and their integration in 3D indicate the performance of the ventilation. Although the injected air velocities do not vary drastically near the duct holes, TDS parameters have been increased by reaching to the end of the duct. The air jet incidences lead to the inclination of the exit flow and change the air distribution pattern. It is nevertheless surprising to show PDDs have a uniform air distribution with the low draft. This feature nominated PDDs as a practical candidate for the large space applications.

According to the condition that is prevailed in this problem, it's noteworthy to evaluate the indoor air quality with vigorous indices. The results show that parameters like EDT underestimate the discomfort zones in comparison to reality. On the other hand, terminal velocities provide satisfactory results.

In brief, the information that came out from the numerical modeling are essential, but not sufficient, for the final design. The 3D contours, besides the local requirements are used by designers to select the best diffuser type and location to minimize the discomfort zone at the occupant zone.

\section{ACKNOWLEDGMENT}

The authors are grateful to the Natural Sciences and Engineering Research Council (NSERC) of Canada for financial support. A part of simulations carried out on a cluster
Mammouth at Centre de Calcul Scientifique, which is operated by the Université de Sherbrooke, QC, Canada. The authors thank the Réseau Québecois de Calcul de Haute Performance and Compute Canada for providing us these computational resources.

\section{REFERENCES}

[1] T. Hjermann, "CFD Simulation of Active Displacement Ventilation", Master Thesis, Norwegian University of Science and Technology (NTNU) - Department of Energy and Process Engineering, 2017.

[2] "Ventilation for Acceptable Indoor Air Quality", ANSI/ASHRAE Standard 62.1, 2016.

[3] Sh.K. Wang, "Handbook of Air Conditioning and Refrigeration", Second Edition, McGraw-Hill, 2001, ISBN 0-07-068167-8.

[4] Ch. Liu, A. Li, Ch. Yang, and W. Zhang, "Simulating Air Distribution and Occupants' Thermal Comfort of Three Ventilation Schemes for Subway Platform", Building and Environment, 125, 15-25, 2017.

[5] B. Sajadi, M.H. Saidi, and A. Mohebbian, "Numerical Investigation of the Swirling Air Diffuser: Parametric Study and Optimization", Energy and Buildings, 43, 1329-1333, 2011

[6] A.k. Melikov, R. Cermak, and M. Majer, "Personalized Ventilation: Evaluation of Different Air Terminal Devices", Energy and Buildings, 34, 829-836, 2002.

[7] A. Fontanini, M. G. Olsen, and B. Ganapathysubramanian, "Thermal Comparison Between Ceiling Diffusers and Fabric Ductwork Diffusers for Green Buildings", Energy and Buildings, 43, 2973-2987, 2011.

[8] "Section E G: Air Distribution Engineering Guide", Price Industries Catalogues, 2011.

[9] Y. Epstein, and D.S. Moran, "Thermal Comfort and The Heat Stress Indices", Industrial Health, 44, 388-398, 2006.

[10] M.A. Aziz, A.M.G. Ibrahim, El.Sh.F.A. Mohammed, and R.H. Mohammed, "Experimental and Numerical Study of Influence of Air Ceiling Diffusers on Room Air Flow Characteristics", Energy and Buildings, 55, 738-746, 2012.

[11] A.P. Gagge, J.A.J. Stolwijk, and J.D. Hardy, "Comfort and Thermal Sensations and Associated Physiological Responses at Various Ambient Temperatures", Environmental Research, 1, 1:1-20 June 1967.

[12] A.F. Alfahaid, "Effects of Ventilation on Human Thermal Comfort in Rooms", Ph.D. Thesis, Old Dominion University, Norfolk, VA, 2000 